\title{
Electro-thermal MEMS fiber scanner for endoscopic optical coherence tomography (Conference Presentation) (Withdrawal Notice)
}

Hyeon-Cheol Park, Xiaoyang Zhang, Jessica MavadiaShukla, Wu Yuan, Huikai Xie, et al.

Hyeon-Cheol Park, Xiaoyang Zhang, Jessica Mavadia-Shukla, Wu Yuan, Huikai Xie, Xingde Li, "Electro-thermal MEMS fiber scanner for endoscopic optical coherence tomography (Conference Presentation) (Withdrawal Notice)," Proc. SPIE 9697, Optical Coherence Tomography and Coherence Domain Optical Methods in Biomedicine XX, 969700 (1 February 2018); doi: 10.1117/12.2214787

SPIE. Event: SPIE BiOS, 2016, San Francisco, California, United States 


\title{
Electro-thermal MEMS fiber scanner for endoscopic optical coherence tomography (Conference Presentation) (Withdrawal Notice)
}

\author{
Proc. SPIE 9697, 969700 (2016)
}

Online Publication Date: 26 April 2016

Withdrawn from Publication: 1 February 2018

Conference Date: 15-17 February 2016

Conference Location: San Francisco, California, United States

Conference Title: Optical Coherence Tomography and Coherence Domain Optical Methods in Biomedicine XX Conference Chairs: Joseph A. Izatt, James G. Fujimoto, Valery V. Tuchin

Hyeon-Cheol Park

Johns Hopkins Univ. (United States)

Publisher's Note: This conference presentation, originally published on 26 April 2016, was withdrawn per author request. 